\title{
The effect of ethyl alcohol on electroencephalographic sleep cycles in cats'
}

\author{
RICHARD B. YULES, JOHN A. OGDEN, FREDERICK P. GAULT AND DANIEL X, FREEDMAN
}

YALE UNIVERSITY SCHOOL OF MEDICINE

EEGs taken from four sleeping cats for three control and four alcohol consecutive nights show that if $1 \mathrm{gm}$ ethanol $/ \mathrm{kg}$ body w'eight is administered $15 \mathrm{~min}$. prior to sleep, Stage 1 REM time dicreases from control values the first two alcohol nights but returns to control levels on the third and fourth alcohol mights. This pattern of change in REM time is the same as that found in humans. The REM time pattern is effected by a change in length rather than in numbers of REM periods. These data support the use of cats in establishing a mechanism for REM sleep which can be extrapolated to humans.

Barbiturates (Oswald et al, 1963), amphetamine (Rechtschaffen \& Maron, 1964), and alcohol (Yules et al, 1966) have been shown to alter EEG sleep stages in man. Alcohol produces an alteration characterized by an initial depression of stage 1 REM (rapid eye movement) sleep which is followed on consecutive nights by a return of REM time to control levels and then increases beyond control levels in the presence of continued drug administration. The effect of these drugs on sleep stages in animals has yet to be assessed. This study was undertaken to determine the effect of ethyl alcohol on consecutive night REM stages of cats, and to compare the pattern of drug effects in man and cat. Method

Four 7-lb. cats (three female; one male) were anesthetized with pentobarbital after which cortical and eye electrodes (Yules et al, 1966) were surgically implanted. After a 10 day anesthesia recovery period, the cats were balanced on a stone surrounded by water, a situation in which they would not REM sleep in order to avoid falling from the stone into the water (W. Dement, personal communication). For five days a sleep-awake cycle was established by placing the cats from their waking stone into recording boxes; control recordings were then begun. Three control nights were followed by four alcohol nights at which time, $15 \mathrm{~min}$. prior to placing the animal in his sleep box, water or $1 \mathrm{gm}$ ethanol per $\mathrm{kg}$ body weight as a $20 \%$ solution was administered by gastric tube. The five cycle, three control and four alcohol nights were all consecutive; animals were fed after their $7 \mathrm{hr}$. sleep period.

EEG tracings were taken on a Grass Model VII polygraph. The per cent of REM time was determined for the total, first half, and second half of the night. The number of REM periods and length was also determined as was the latency or time to the first REM period.

Results

As shown in Fig. 1 and Table 1, if the mean per cent REM time of three control nights for all four cats is

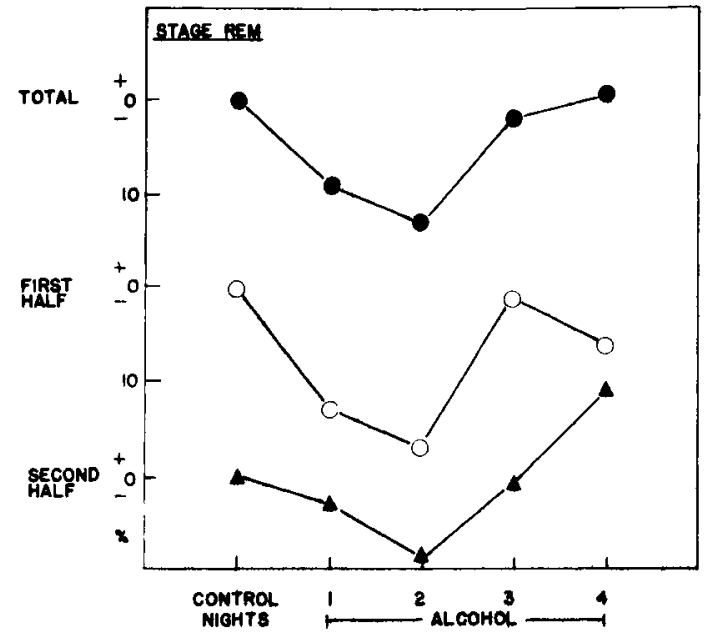

Fig. 1. The pattern of change of mean (four cats) percentages for 4 consecutive alcohol nights compared to 3 control nights. $0 \%$ represents for the total, first half, and second half night respectively $30 \%, 32 \%$, and $29 \%$ for Stage 1 REM. Raw data for stage 1 REM from which the points were plotted are presented in Table 1.

taken as a 0 point of reference, during four consecutive alcohol nights, stage 1 REM decreases below control levels on the first two alcohol nights but climbs back to control levels on the third and fourth alcohol nights. The changes occur more prominently in the first half of the night, but do occur, albeit to a lesser degree, during the second half of the night. As shown in Fig. 2, the pattern of change seen in REM time is reflected in the length of each REM period (Table 2) and not in the number of REM periods which vary unsystematically by no more than 5 REM periods from the control level of 18 REM periods. Latency periods vary randomly on alcohol nights compared to control latency periods (Table 3). The changes described above occurred in each cat. The REM time of all cats is decreased by $8-14 \%$ on the first two alcohol nights, a decrease in length per REM perlod of $2 \mathrm{~min}$. Under systematic doses of alcohol, no further decrease in REM time is seen.

Table 1. Mean REM Time for Four Cats (per cent)

\begin{tabular}{lrlllllllll} 
Nights: & \multicolumn{1}{c}{ Control* } & \multicolumn{1}{c}{ Alcohol } \\
\hline Stoge 1 REM & Mean & SD & 1 & SD & 2 & SD & 3 & SD & 4 & SD \\
\hline Total & 30 & 2.0 & 21 & 8.5 & 17 & 3.3 & 28 & 5.5 & 31 & 6.5 \\
First-half & 32 & 3.0 & 17 & 9.3 & 13 & 6.0 & 29 & 7.0 & 24 & 7.0 \\
Second-half & 29 & 2.0 & 26 & 8.2 & 21 & 6.2 & 28 & 4.8 & 39 & 6.7 \\
\hline
\end{tabular}

* Mean of three nights 


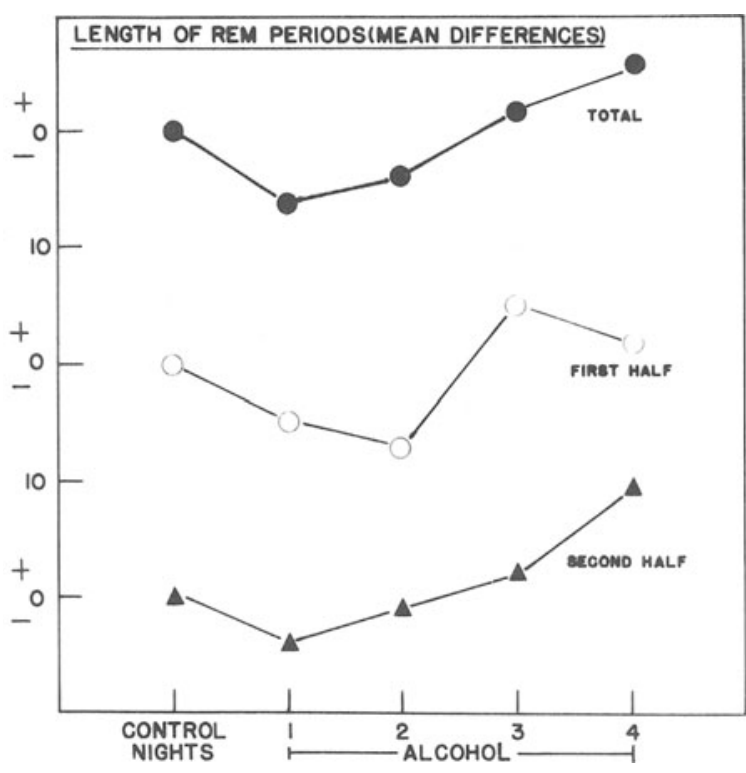

Fig. 2. The pattern of change of means (four cats) for 4 consecutive alcohol nights compared to 3 control nights. 0 represents for the total, first half, and second half night respectively 21,21 , and 18 page long REM periods. The length/REM period point in seconds $\times 10$ is presented in Table 2 . Note how closely the length/ REN period graph follows the \% of REM time graph in Fig. 1.

\section{Discussion}

REM time decreases from control levels the first two alcohol nights but returns to control levels on the fourth alcohol night. These changes occur in both halves of the night, but are more pronounced during the first half of the night when alcohol concentrations must be at highest levels (Harger, 1937).

The mechanism by which the pattern of REM is effected is concerned not with a change in number of periods of REM but rather with a change in length of REM periods. In view of Jouvet's ability to change the timing of REM periods by electrical stimulation of mesencephalic reticular structures as well as to elimi-

Table 2. Length / REM Period (minutes)

\begin{tabular}{lcccccccc} 
& \multicolumn{3}{c}{ Control } & & \multicolumn{3}{c}{ Alcohol } \\
\hline Nights: & 1 & 2 & 3 & 1 & 2 & 3 & 4 \\
\hline total & 8 & 7 & 7 & 5 & 6 & 8 & 9 \\
first half & 8 & 8 & 7 & 6 & 5 & 9 & 8 \\
second half & 7 & 7 & 6 & 5 & 6 & 7 & 9 \\
\hline
\end{tabular}

Table 3. Latency (minutes to first REM period)

\begin{tabular}{lrrrrrrr} 
Nights: & 1 & 2 & 3 & 1 & 2 & 3 & 4 \\
\hline Cat 1 & 20 & 52 & 34 & 8 & 30 & 12 & $*$ \\
Cat 2 & 20 & 25 & 25 & 30 & 84 & 9 & 23 \\
Cat 3 & 42 & 46 & 49 & 57 & 77 & 93 & 97 \\
Cat 4 & 27 & 106 & 43 & 36 & 87 & 34 & 50 \\
\hline
\end{tabular}

* No precise measure obtained because of temporary failure of electrode channel of eye.

nate REM periods by electrocoagulating the R.P.Nucleus (Jouvet, 1964), these areas should be considered as a possible site of action of alcohol.

The effect of alcohol on cat EEG sleep patterns is similar to the effect on human sleep patterns. Cat and human REM time follow the same pattern for the four alcohol nights studied here. Whether cat REM time increases above control levels on later alcohol nights and on recovery nights as it does in humans is yet to be determined. Yet it is clear that alcohol acts to initially decrease REM time in both cats and humans, an action which may be mediated through similar mechanisms.

Since alcohol effects REM time similarly in cats and humans, experiments are now in order to determine the sites of action of drugs in cats, results which combined with Jouvet's data (Jouvet, 1964) on nuclei controlling REM sleep may permit a more accurate statement of human sleep mechє nisms.

\section{References}

Harger, R. N., Hulpieu, H. R., \& Lamb, E. G. The speed with which various parts of the body reach equillibrium in the storage of ethyl alcohol. J. biol. Chem., 1937, 120, 689-704.

Jouvet, $M$. Sleep in pontine cats: studies on the mechanisms of rhombencephalic phase of sleep. EEG clin. Neurophysiol., 1964 17,446 .

Oswald, I., Berger, R. J., Jaramillo, R. A., Keddie, R. I. G. Olley, P. C., \& Plumkett, G. B. Melancholia and barbiturates: a controlled EEG, body and eye movement study of sleep. Brit. J. Psychiat., 1963, 109, 66-78.

Rechtschaffen, A., \& Maron, L. The effects of amphetamine on the sleep cycle. EEG clin. Neurophysiol., 1964, 16, 438-445.

Yules, R. B., Ogden, J. A., Gault, F. P., \& Freedman, D. X. A surgically implanted electrode for recording both rapid eye movements and muscle tone during sleep. EEG clin. Neurophysiol., 1966, 20, 410-411.

Yules, R. B., Freedman, D. X. \& Chandler, K. A. The effect of ethyl alcohol on man's electroencephalographic sleep cycle. EEG clin. Neurophysiol., 1966, 20, 109-111.

\section{Note}

1. Research supported by USPHS MH 0705, USPHS K3-18566 and M1463. 